\title{
A Novel Whisker Sensor Used for 3D Contact Point Determination and Contour Extraction
}

\author{
Hannah M. Emnett ${ }^{1}$, Matthew M. Graff ${ }^{1}$, Mitra J. Z. Hartmann ${ }^{1,2}$ \\ ${ }^{1}$ Department of Mechanical Engineering, ${ }^{2}$ Department of Biomedical Engineering \\ Northwestern University, Evanston, IL 60208
}

\begin{abstract}
We developed a novel whisker-follicle sensor that measures three mechanical signals at the whisker base. The first two signals are closely related to the two bending moments, and the third is an approximation to the axial force. Previous simulation studies have shown that these three signals are sufficient to determine the three-dimensional (3D) location at which the whisker makes contact with an object. Here we demonstrate hardware implementation of 3D contact point determination and then use continuous sweeps of the whisker to show proof-of principle 3D contour extraction. We begin by using simulations to confirm the uniqueness of the mapping between the mechanical signals at the whisker base and the 3D contact point location for the specific dimensions of the hardware whisker. Multi-output random forest regression is then used to predict the contact point locations of objects based on observed mechanical signals. When calibrated to the simulated data, signals from the hardware whisker can correctly predict contact point locations to within 1.5 cm about $74 \%$ of the time. However, if normalized output voltages from the hardware whiskers are used to train the algorithm (without calibrating to simulation), predictions improve to within $1.5 \mathrm{~cm}$ for about $96 \%$ of contact points and to within $0.6 \mathrm{~cm}$ for about $78 \%$ of contact points. This improvement suggests that as long as three appropriate predictor signals are chosen, calibrating to simulations may not be required. The sensor was next used to perform contour extraction on a cylinder and a cone. We show that basic contour extraction can be obtained with just two sweeps of the sensor. With further sweeps, it is expected that full 3D shape reconstruction could be achieved.
\end{abstract}

Keywords: vibrissa, robot, active sensing, tactile, touch, trigeminal

\section{INTRODUCTION}

Although primates, including humans, rely strongly on their visual systems to explore the world, most mammals inhabit a world of touch and scent. In the case of rodents, whiskers are a particularly important source of touch information [1-3]. Whiskers, also called "vibrissae," are long stiff hairs, arranged in regular rows and columns, protruding from the rodent's cheek [4].

There are no sensors along the length of a whisker, instead, all sensing is performed by mechanoreceptors densely packed within a follicle at the whisker base [5]. The whiskers are thus essentially flexible cantilever beams. Under quasistatic, frictionless conditions, three forces and three moments (torques) at the whisker base are sufficient to describe all mechanical information entering the follicle. A recent study found that several - but not all - triplet combinations of these six mechanical signals are sufficient to uniquely determine the 3D point at which a whisker has made contact with an object [6]. In principle, these simulation results suggest that sensing an appropriate triplet of mechanical signals could permit an artificial (robotic) whisker to be used for 3D contact point determination and thus feature extraction.

In the present work, we developed a biomimetic model of a rat whisker-follicle complex for use in 3D feature extraction. We specifically focused on creating a whisker with appropriate material properties and taper, as well designing a follicle that can measure the specific mechanical signals requisite for contact point determination.

Several previous studies have used rates of change of bending moment to determine contact point location, specifically to achieve the radial distance from the base of the follicle [7--14]. The novelty of the present work lies in achieving an alternate method for contact point determination that does not rely on time rates of change of mechanical signals. Instead, triplets of mechanical signals are used at each instant in time to estimate the 3D coordinates of whisker-object contact.

Results are discussed in the context of using whiskerbased tactile systems to provide spatial mappings in partiallyoccluded spaces, murky waters, or sand, and as sensors that can be recalibrated remotely in real time for use in underwater exploration and other remote sensing applications.

\section{Methods}

\section{A. Artificial Whisker}

A photograph of a biological rat whisker is shown in Fig. 11a). From outside to in, the whisker consists of the cuticle, the cortex, and the medulla, which can be approximated as hollow [15-18]. Whiskers have an intrinsic curvature that is approximately quadratic and a Young's modulus that ranges between $2.90 \mathrm{GPa}$ to $4.92 \mathrm{GPa}$ and also increases slightly from base to tip [17, 19, 20]. Rat whiskers have an arc length (S) that typically ranges between 7-50 $\mathrm{mm}$, a base diameter $\left(D_{\text {Base }}\right)$ between 50-100 microns, and a tip diameter $\left(D_{T i p}\right)$ between 1-5 microns, although the tip diameter can vary greatly due to damage, wear, and barbering. All rat whiskers taper approximately linearly from base to tip [21-24]. The taper of a whisker is defined by its diameter slope, $\left(D_{\text {Base }}-D_{\text {Tip }}\right) / S$, which ranges from 0.00208 and 0.0139 (median 0.00407) and a base/tip diameter ratio, $\left(D_{\text {Base }} / D_{\text {Tip }}\right)$, which is much more variable ranging between 2 and 148 (median 32) [25].

In the present work, we simplified the biological whisker to create a solid, straight, linearly-tapered artificial whisker, as schematized in Fig. 1 (b). Solid whiskers are easy to manufacture and straight, tapered whiskers have previously been shown to have a unique mapping between mechanical signals and 3D contact point [6]. This simplified model has 
only three geometric parameters of interest $\left(D_{\text {Base }}, D_{\text {Tip }}\right.$, and $S$ ). We used a plastic whisker with a constant Young's modulus along the length. The whisker had a base diameter of $1.67 \mathrm{~mm}$, a length of $195 \mathrm{~mm}$, and tapered linearly to a tip diameter of $0.47 \mathrm{~mm}$. These dimensions yielded a diameter slope of 0.00615 and a base/tip diameter ratio of 3.55.

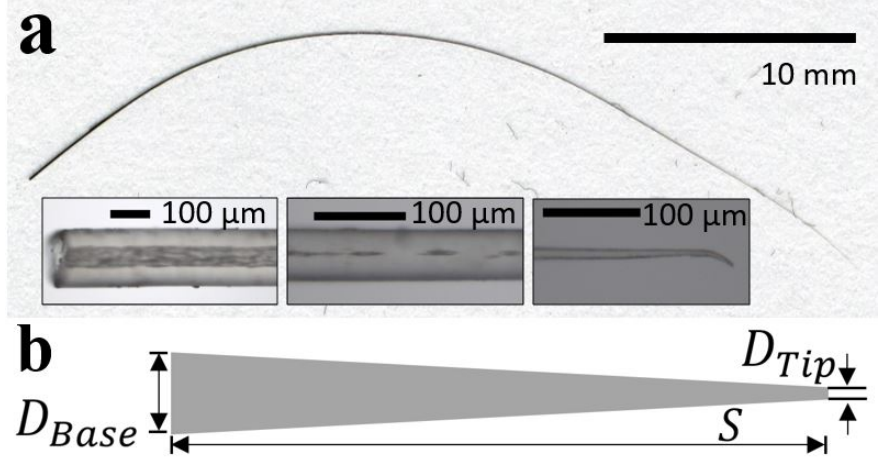

Fig. 1. Biological whisker compared with the artificial whisker used in the present experiments: (a) From outside to in, the biological rat whisker consists of cuticle, cortex, and medulla. The three insets illustrate these whisker components at the base (x10 magnification), middle (x20 magnification), and tip (x20 magnification) of the whisker. The insets and main image were obtained from four different whiskers. The main image was scanned with an Epson 4180 scanner at $4800 \mathrm{dpi}$ and the insets were taken with an Olympus BX60 microscope and a MBF Bioscience Stereo Investigator DV-47 color camera. (b) The simplified artificial whisker used in the present study lacked intrinsic curvature and neglected the effects of the medulla and cuticle. The relevant geometric parameters therefore included only $D_{\text {Base }}, D_{\text {Tip }}$, and $S$. The schematic is not to scale.

\section{B. Artificial follicle and signal acquisition}

As will be described in Sec. IIII. we designed and constructed a novel follicle-like sensor to measure the mechanical signals at the base of the artificial whisker. The follicle used a Maltese cross configuration with four arms. Each arm of the cross had a pair of strain gages (top and bottom) that completed a half Wheatstone bridge tuned with a potentiometer into the desired voltage range. There were four Wheatstone bridges, one for each arm, so each arm is associated with a single voltage, denoted $V_{1}, V_{2}, V_{3}, V_{4}$.

Voltages were amplified with a gain of 1,000 (INA129P, National Instruments, USA) and filtered at $1.5 \mathrm{kHz}$ before being digitally acquired with a 16-bit delta-sigma analog-todigital converter (ADS1178, Texas Instruments, USA) with a modulator frequency of $3.4 \mathrm{MHz}$ and an output data rate of $500 \mathrm{~Hz}$. A PIC32 microcontroller (PIC32MX795F512H, Microchip, USA) communicating over a serial peripheral interface (SPI) acquired the data from the converter. The PIC32 then forwarded the data to MATLAB $\cap$ over USB.

\section{Experimental measurements of whisker deflections}

Our goal during experiments was to investigate the relationship between mechanical signals at the whisker base and the location of the 3D whisker-object contact point. Six mechanical signals are generated at the base of a whisker when it deflects. $F_{Y}$ and $F_{Z}$ are the transverse forces, $M_{Z}$ and $M_{Y}$ are the associated bending moments, $F_{X}$ is the axial force (along the length of the whisker), and $M_{X}$ is the twisting moment about the whisker's own axis. Three of these signals, $M_{Y}, M_{Z}$, and $F_{X}$, are schematized in Fig. 2(a) as the target signals to be measured by the sensor.

We used a custom-built pegboard (Fig. 2(b) and Fig. 2(c) to perform experiments that could directly relate mechanical signals at the sensor base to the 3D contact point location. The coordinates of the contact point ("cp") are denoted as $r_{c p}$, $\theta_{c p}$, and $\phi_{c p}$. This coordinate system is identical to a standard spherical coordinate system except that $\phi_{c p}$ is measured from the $x-y$ plane instead of from the z-axis.

During experiments, the whisker was manually deflected to several different 3D positions on the pegboard. The distance $r_{c p}$ was varied between $3.0-5.0$ inches $(7.62-12.7 \mathrm{~cm})$ in 1.0 inch $(2.54 \mathrm{~cm})$ increments (3 values). The angle $\theta_{c p}$ was varied between $\pm 20^{\circ}$ in $5^{\circ}$ increments ( 8 values excluding $\left.0^{\circ}\right)$. Elevation was varied between \pm 1.0 inches $(2.54 \mathrm{~cm})$ in 0.25 inch $(0.635 \mathrm{~cm})$ increments (8 values excluding zero elevation). This procedure yielded a total of $3 * 8 * 8=$ 192 whisker deflections. In all analyses, the elevation distance was converted to an angle, $\phi_{c p}$. During data collection, we alternated directions in both $\theta_{c p}$ and elevation so as to mitigate any possible effects of mechanical hysteresis in the whisker material and sensor.

The signals $V_{1}$ to $V_{4}$ were centered and normalized before analysis. For each signal, we recorded data for $200 \mathrm{msec}$ while the whisker was at rest. These data were averaged and subtracted from all subsequent trials in order to center the signals around zero. Then, each of the four voltages was normalized between -1 and 1 over the full range of experimental deflections in order to account for any differences in sensitivity between arms of the cross.

\section{Computing predictor signals based on voltage measure- ments from the follicle}

Each arm of the Maltese cross of the artificial follicle output a single voltage $\left(V_{1}\right.$ to $\left.V_{4}\right)$. By considering the degrees of freedom involved, it can be shown that a minimum of three predictor signals is required to uniquely predict 3D contact point location. Previous simulation work has specifically shown that the triplet $\left[M_{B}, M_{D}, F_{X}\right]$ is one of several functional triplets for estimating contact point locations. Using this triplet as inspiration, we searched to find three predictors that use hardware signals for contact point estimation.

Equations 1 [ 5 show the calculations to obtain the predictors used in the present work. Predictors 1 and 2 are closely related to $M_{B}$ and $M_{D}$. Mechanically, we expected $F_{X}$ to be the sum of $V_{1}$ to $V_{4}$, however, we found that the magnitude of this signal was outside the range of the sensitivity of our sensor. We therefore empirically chose a new predictor, $P_{3}$, equal to the sum of the absolute value of all four voltages. Although $P_{3}$ is not mechanically equivalent to $F_{X}$, its general behavior as the whisker is deflected was empirically found to resemble expected (simulated) values for $F_{X}$. 

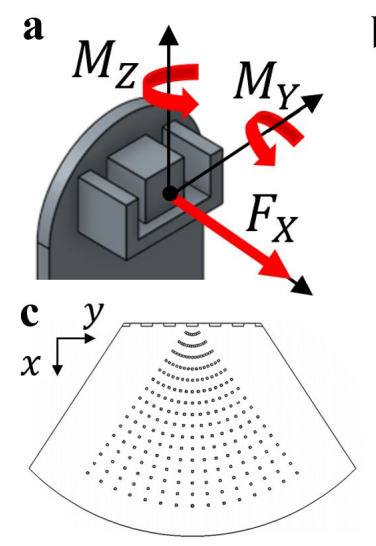

b

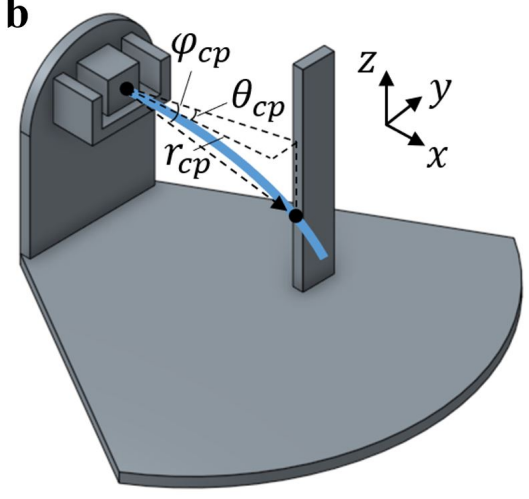

Fig. 2. Mechanical signals at the whisker base and experimental setup: (a) Whisker deflection generates three forces and three moments at the whisker base. Our system specifically targeted the two bending moments $\left(M_{Y}\right.$ and $M_{Z}$ ) as well as the axial force $\left(F_{X}\right)$. (b) A pegboard was constructed to place the hardware whisker systematically and reliably at different 3D contact point locations. In each trial, the whisker was deflected to a contact point defined by $r_{c p}, \theta_{c p}$, and the vertical distance (z). The vertical ruler had notches on the side to hold the whisker in place at a single point. The radial distance $r_{c p}$ is measured as the straight-line distance from the base of the whisker to the contact point. The horizontal angle $\theta_{c p}$ is the angular deflection in the $x-y$ plane. The vertical distance is the straight-line distance from the whisker base to the z-location of the contact point and was later converted to $\phi_{c p}$, the angle between the contact point and the $x-y$ plane. (c) The two-dimensional (2D) schematic illustrates a top-down view of the increments on the pegboard. Spacing is 0.5 inch $(1.27 \mathrm{~cm})$ in the radial dimension, and $5^{\circ}$ increments for deflection in the $\mathrm{x}-\mathrm{y}$ plane $\left(\theta_{c p}\right)$. Not shown is the vertical ruler that held the whisker at 0.25 in $(0.635 \mathrm{~cm})$ elevation increments.

$$
\begin{gathered}
M_{Y}=V_{1}-V_{2} \\
M_{Z}=V_{3}-V_{4} \\
P_{1} \approx M_{B}=\sqrt{M_{Y}^{2}+M_{Z}^{2}} \\
P_{2} \approx M_{D}=\arctan \frac{M_{Z}}{M_{Y}} \\
P_{3}=\left|V_{1}\right|+\left|V_{2}\right|+\left|V_{3}\right|+\left|V_{4}\right|
\end{gathered}
$$

The signal $P_{1}$ was normalized between 0 and 1 , the signal $P_{2}$ was normalized between -1 and 1 , and the signal $P_{3}$ was normalized between -1 and 0 .

\section{E. Simulations of whisker deflections}

In portions of Sec. III] experimental signals obtained from the hardware whisker were compared with signals predicted by simulated whisker deflections. We used a model for quasistatic whisker bending to perform these simulations [6]. The whisker was simulated to have the same dimensions as the experimental whisker, and Young's modulus was set to 3.0 GPa.

The whisker was simulated to deflect through the identical range as the experiments, but at a much higher resolution. Thus, during simulations, the whisker was deflected in $r_{c p}$ between $7.62 \mathrm{~cm}$ and $12.7 \mathrm{~cm}$ in increments of $2.54 \mathrm{~cm}(3$ values), between $\pm 25^{\circ}$ in $1^{\circ}$ increments for $\theta_{c p}$ (51 values) and between $\pm 25^{\circ}$ in $1^{\circ}$ increments for $\phi_{c p}$ (51 values). This procedure yielded a total of $3 * 51 * 51=7,803$ unique whisker deflections. For 303 unique contact points in the full simulation dataset, the optimization procedure could not find a solution. These deflections were on the edges of the space and were excluded from the full dataset. The final simulation dataset therefore contained 7,500 unique values. In these simulations, the whisker was deflected to a simulated contact point $\left[r_{\text {cpsim }}\right.$, $\left.\theta_{\text {cpsim }}, \phi_{\text {cpsim }}\right]$ and the output was the mechanical signals $M_{Y s i m}, M_{Z s i m}$, and $F_{X s i m}$. As will be described below, we subsequently used Random Forest regression to generate the inverse mapping which uses mechanical signals as inputs and computes the contact point as output.

\section{F. Calibrating experimental data to match simulations}

In Fig. 4(b) and 4(c) of Sec. III] we assessed the ability of the artificial whisker to match the simulated data using only a linear calibration. The idea here is that if the hardware follicle can be forced to match simulation, it should in principle achieve the same mapping uniqueness as the simulations. The calibration coefficients $C_{1}-C_{8}$ were found as shown in eq. 6

$$
\left[\begin{array}{cccc}
C_{1} & -C_{2} & 0 & 0 \\
0 & 0 & C_{3} & -C_{4} \\
C_{5} & C_{6} & C_{7} & C_{8}
\end{array}\right]=\left[\begin{array}{c}
M_{Y \text { sim }} \\
M_{Z s i m} \\
F_{X s i m}
\end{array}\right]\left[\begin{array}{l}
V_{1} \\
V_{2} \\
V_{3} \\
V_{4}
\end{array}\right]^{-1}
$$

For ease of calculation, the bending magnitude and direction were used in their component forms $M_{Y}$ and $M_{Z}$. The signals $M_{Y \text { sim }}, M_{Z \text { sim }}$, and $F_{X \text { sim }}$ are generated in simulation, the voltages $\left(V_{1}\right.$ to $\left.V_{4}\right)$ are experimentally measured, and the calibration coefficients $\left(C_{1}-C_{8}\right)$ are computed. We then use $C_{1}-C_{8}$ to calibrate the experimental voltages, as shown in eq. 7. to generate $M_{Y c a l}, M_{Z c a l}$, and $F_{X c a l}$.

$$
\left[\begin{array}{c}
M_{Y c a l} \\
M_{Z c a l} \\
F_{X c a l}
\end{array}\right]=\left[\begin{array}{cccc}
C_{1} & -C_{2} & 0 & 0 \\
0 & 0 & C_{3} & -C_{4} \\
C_{5} & C_{6} & C_{7} & C_{8}
\end{array}\right]\left[\begin{array}{l}
V_{1} \\
V_{2} \\
V_{3} \\
V_{4}
\end{array}\right]
$$

Simulated $M_{B}$ and $M_{D}\left(M_{B \text { sim }}\right.$ and $\left.M_{D \text { sim }}\right)$ were then computed from $M_{Y \text { sim }}$ and $M_{Z \text { sim }}$, and calibrated $M_{B}$ and $M_{D}\left(M_{B c a l}\right.$ and $\left.M_{D c a l}\right)$ were computed from $M_{Y c a l}$ and $M_{Z c a l}$. The signals $M_{B s i m}$ and $M_{B c a l}$ were normalized between 0 and 1, the signals $M_{D \text { sim }}$ and $M_{D c a l}$ were normalized between -1 and 1 , and the signals $F_{X \text { sim }}$ and $F_{X c a l}$ were normalized between -1 and 0 .

\section{G. Generating the mappings between mechanical signals and the $3 D$ contact point}

The mappings between mechanical signals at the whisker base and the 3D whisker-object contact point are extremely non-linear. We used the scikit-learn implementation of Random Forest Regression function in Python to generate these mappings, with maximum tree depth limited to $30 \%$ of the length of the input data and training set size set to $95 \%$ of the data. Mean square error was used to evaluate the quality of each split and 10-fold cross-validation was used to evaluate each mapping after fitting. Termination criterion was reached when the coefficient of determination for $r_{c p}$ in the test set was greater than 0.93 . The radial contact point was chosen as the termination criterion because it is the most difficult of the three spherical coordinates to predict. All $r^{2}$ values 
reported are evaluated over the entire experimental dataset. The mappings were generated in two slightly different ways in different portions of Sec. III The mappings in Fig. 4 (a) and Fig. 4(c) are essentially used as a "look-up table." In contrast, Fig. 4(d), and Fig. 5 use the Random Forest mappings in a predictive manner, with distinct training and test sets.

The analysis of Fig. 4(a) uses purely simulation results to confirm that the whisker possesses a unique mapping between mechanical signals and the 3D contact point. The complete set of mechanical signals obtained at all 7,500 deflection points was used as input to the random forest regression. There is no "training" or "test" set because the goal of the analysis was to generate the mapping and to test for mapping uniqueness. The mapping is essentially a "look-up table" between $\left[M_{B s i m}, M_{D s i m}, F_{X s i m}\right]$ and output $\left[r_{c p}, \theta_{c p}, \phi_{c p}\right]$. The cross-validation score during the mapping was 1.0.

The analysis of Fig. 4(c) uses a subset of the data used to generate Fig. 4(a) that matches the experimental contact point locations. This analysis tests $3 \mathrm{D}$ contact point estimation when experimental mechanical signals have been calibrated to those obtained through simulation. The inputs were $\left[M_{B c a l}\right.$, $\left.M_{D c a l}, F_{X c a l}\right]$ and the outputs were $\left[r_{c p}, \theta_{c p}, \phi_{c p}\right]$. The cross-validation score during the mapping was 0.94 and the coefficients of determination were all above 0.99. Again, there is no training and test set here; the Random Forest is being used only to generate a look-up table for the mapping.

Finally, the analysis of Fig. 4(d) and Fig. 5 relies only on mappings generated using purely experimental data. No simulations are used.

\section{H. Acquiring data during a continuous whisker sweep against an object}

To test the ability of the mapping to extract a continuous 3D contour, we designed an experimental set-up that allowed us to rotate the whisker-follicle sensor in one dimension. During tests of the sensor (Fig. 5), the follicle-whisker system was fixed so that rotation was allowed only about the z-axis. Two shapes - a cylinder and a cone - were placed in front of the whisker. The cylinder had a radius of $4.5 \mathrm{~cm}$ and the cone had a base radius of $2.6 \mathrm{~cm}$ and height of $6 \mathrm{~cm}$. The radial contact point changed as the whisker swept against the object but was located approximately $12 \mathrm{~cm}$ from the whisker base at the start of each trial. The whisker was $\sim 19.5 \mathrm{~cm}$ long, so $12 \mathrm{~cm}$ is approximately $60 \%$ out along the whisker length.

Voltage data were acquired continuously as the whisker was rotated against each shape. Once at full deflection, data collection was stopped, the whisker was returned to neutral and another sweep began. The "contact" portions of the sweep were established by thresholding. Contact was determined to have begun when the average absolute value of the normalized voltages across all four arms was above $35 \%$ of the maximum and stopped when the voltage decreased below $0.99 \%$ of the maximum. The voltage recorded during the first sample of the sweep was subtracted from all subsequent voltages in order to center the data around zero. Each of the four sensor voltage outputs was normalized between -1 and 1 using the full range of the data obtained in the pegboard experiments. A moving average filter with a window size of three was used to digitally smooth the data.

An encoder at the base of the whisker was used to measure rotation of the whisker. Encoder resolution was 448 counts/revolution and signals were read over SPI at $200 \mathrm{~Hz}$. Linear interpolation was used to approximate the whisker rotation angle for each voltage output of the sensor. Information from the encoder was used only to convert the 3D contact points from whisker-centered coordinates to the laboratory frame. No information from the encoder was used to determine where contact occurred along the whisker length. This choice was made to reflect the fact that rats have very few proprioceptors in the whisker muscles [26].

The 3D contact point locations were predicted using a random forest regression algorithm trained on the data obtained in the pegboard experiments. To convert to the laboratory frame, the predicted $3 \mathrm{D}$ contact point locations were converted to Cartesian coordinates, incorporating the encoder angle, according to eq. 8 where "enc" denotes "encoder."

$$
\left[\begin{array}{l}
x \\
y \\
z
\end{array}\right]=\left[\begin{array}{ccc}
\cos \left(\theta_{\text {enc }}\right) & -\sin \left(\theta_{\text {enc }}\right) & 0 \\
\sin \left(\theta_{\text {enc }}\right) & \cos \left(\theta_{\text {enc }}\right) & 0 \\
0 & 0 & 1
\end{array}\right]\left[\begin{array}{c}
r_{c p} \cos \left(\theta_{c p}\right) \\
r_{c p} \sin \left(\theta_{c p}\right) \\
r_{c p} \tan \left(\phi_{c p}\right)
\end{array}\right]
$$

\section{RESULTS}

We first describe a novel follicle design that can measure three relevant signals at the whisker base. We next explore two separate methods for $3 \mathrm{D}$ contact point determination. In the first method, we perform contact point determination after explicitly calibrating the output of the robotic whisker to match simulation results. This method offers only moderate quality results for large angles of deflection. Therefore, in a second approach, we performed 3D contact point determination using the normalized voltage output directly from the strain gages. Finally, we use the second method to perform continuous 3D contour extraction while sweeping the whisker against two different objects.

\section{A. Follicle design}

The follicle was based on a Maltese cross configuration (Fig. 3[a)), similar to [27] and [28]. Eight strain gages (120 $\Omega, 1.5$ $\mathrm{mm}$ grid, Omega, USA) were affixed, two to each arm, top and bottom, of $0.3 \mathrm{~mm}$ formable 3003 aluminum sheet is water jet cut into a $12.7 \mathrm{~mm}(0.5 \mathrm{inch})$ cross. Each pair of strain gages (one arm of the cross) completes half of a Wheatstone bridge. The plastic whisker was threaded directly into a $12.7 \mathrm{~mm}$ (0.5 inch) long hexagonal "stand-off" attached to the center of the aluminum cross by a M0x80 bolt. When the whisker is deflected (Fig. 3(b)) it bends and causes the opposing strain gages on each arm to be under tension and compression. This will cause an overall change of voltage measurable with the Wheatstone bridge.

To assemble the follicle, the cross was held in place using four M0x80 bolts and custom clamps 3D printed on a Form 
2 3D printer (FormLabs, USA) (Fig. 3(c)). A photograph of a complete follicle-whisker complex is shown in Fig. 3(d). All experiments described in the following sections used a folliclewhisker complex similar but not identical to that shown in Fig. 3(d) with geometric parameters of $S=19.5 \mathrm{~cm}, D_{\text {Base }}=1.67$ $\mathrm{mm}, D_{\text {Tip }}=0.47 \mathrm{~mm}$.

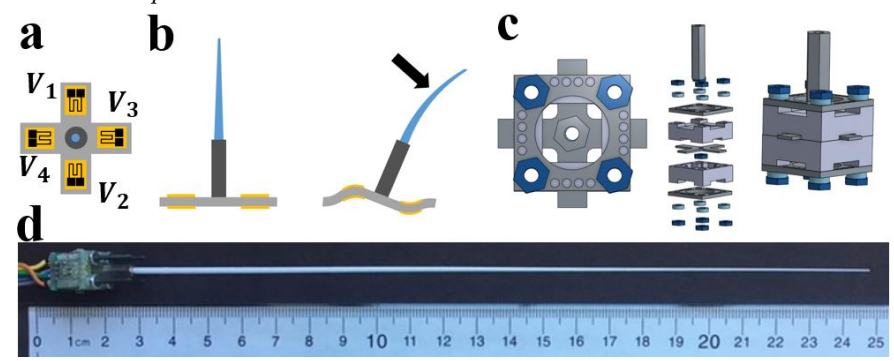

Fig. 3. Design for an artificial follicle: (a) Top view of a schematic of the cross with strain gages affixed. The labels $V_{1}$ to $V_{4}$ denote the voltages obtained from the follicle. (b) Side-view of the cross and whisker when stationary (left) and after a deflection in one direction (right). (c) 3D drawings of the follicle assembly (d) Example picture of a fully assembled follicle. The whisker used in experiments was slightly shorter, with a total length of 19.5 $\mathrm{cm}$

B. Simulations demonstrated a unique relationship between $M_{B}, M_{D}$, and $F_{X}$ and the $3 D$ location of the whiskerobject contact point

We began by confirming that the mappings from mechanics to $3 \mathrm{D}$ contact point were unique for a whisker with our chosen dimensions, as suggested in Huet et al., 2017. To do this, we simulated a whisker with approximately the same geometry and Young's modulus as the hardware whisker. The whisker was deflected to a total of 7,500 unique contact point locations and the uniqueness was evaluated with Random Forest Regression (see Sec. II). Results of these simulations are shown in Fig. 4 (a). The three subplots in this figure show the experimental contact point coordinate known from simulations (either $r_{c p}, \theta_{c p}$, or $\phi_{c p}$ ) versus the predicted contact point coordinate. If the mapping were not unique, each plot would show increased variance at each contact point location and a lower coefficient of determination. Instead, the graphs indicate very tight linearity, with coefficient of determinations indistinguishable from 1.0 for all three contact point coordinates. These high correlations indicate that the geometry we chose for the hardware whisker is adequate to obtain a unique mapping between $\left[M_{B}, M_{D}, F_{X}\right]$ and $\left[r_{c p}\right.$, $\left.\theta_{c p}, \phi_{c p}\right]$.

C. A linear calibration approximately matches voltage outputs to simulated mechanical signals

The simulation results shown in Fig. 4(a) give confidence that - if we were able to make the hardware outputs match those of simulations - then our predictors for the hardware whiskers should be $M_{B}, M_{D}$, and $F_{X}$ at the whisker base to uniquely determine $r_{c p}, \theta_{c p}$, and $\phi_{c p}$.

However, many different types of unpredictable nonlinearities might be present in hardware. We therefore tested the extent to which a linear calibration could force the hardware outputs to match those from simulation. Specifically, we computed $M_{B c a l}, M_{D c a l}$, and $F_{X c a l}$ using eqs. 6 and 7 . Fig. 4 (b) demonstrates that the linear calibration was generally, but not completely, sufficient to force the experimental voltages to match those predicted in simulation.

As indicated previously, the resolution of our sensor was too low to measure $F_{X}$ directly, so we chose the third predictor $\left(P_{3}\right)$ based on the empirical finding that its general trend resembled the axial force signal obtained from simulation. Unsurprisingly, as shown in the bottom panel of Fig. 4(b), the axial force $\left(P_{3}\right)$ showed the largest deviation from simulation results.

The second predictor $\left(P_{2}\right.$, bending direction) exhibited the smallest error of the three signals, and nearly perfectly matched simulation results. Both bending magnitude and axial force $\left(P_{1}\right.$ and $\left.P_{3}\right)$ exhibited the largest errors when signals were the largest, which tended to occur for proximal contacts. These results suggest that contact point estimation will tend to improve for more distal contacts.

\section{D. $3 D$ contact point estimation with experimental data lin-} early calibrated to match simulation results

We compared a subset of the simulated data that had 3D contact points corresponding to the experimental contact points to the calibrated experimental data. We used a lookup table generated by a random forest regression tree on the simulated dataset to estimate contact point location for the calibrated experimental data. Because the hardware whisker had been calibrated to match simulation (Fig. 4(b)), we expected a good match. However, although both $\theta_{c p}$ and $\phi_{c p}$ show $r^{2}$ values above 0.94 , the radial contact point has an $r^{2}$ value of only 0.67 .

Further analysis showed that $74 \%$ percent of the calibrated data (143 out of 192 data points) predicted the Euclidean distance to within $1.5 \mathrm{~cm}$ of the actual contact point and $82 \%$ (157 out of 192 data points) predicted the distance to within $2.5 \mathrm{~cm}$. Two conditions were associated with particularly large errors: small angle deflections and proximal radial contacts. Of the 49 predictions that had errors greater than $2.5 \mathrm{~cm}, 48$ were deflections through angles less than $10^{\circ}$, and 33 were for angles less than $5^{\circ}$.

Therefore, the black points in Fig. 4(c) show the result of predicting the contact point location while excluding any contact points with angles less than $10^{\circ}$, with excluded points plotted in red. These excluded points had the largest effect on the predictions for radial distance and are likely due to errors in the approximation associated with $P_{3}$. Figure 4(c) also clearly shows that while the excluded (small angle) points in the $\theta_{c p}$ and $\phi_{c p}$ subplots are clustered around zero, larger errors occurred at small radial distances. No data obtained for the most distal radial distance tested $(12 \mathrm{~cm})$ had an error greater than $1.5 \mathrm{~cm}$. This finding parallels the results shown in Fig. 4(b), in which the variance increases with larger values of $P_{1}$ and $P_{3}$ (those near the whisker base). 


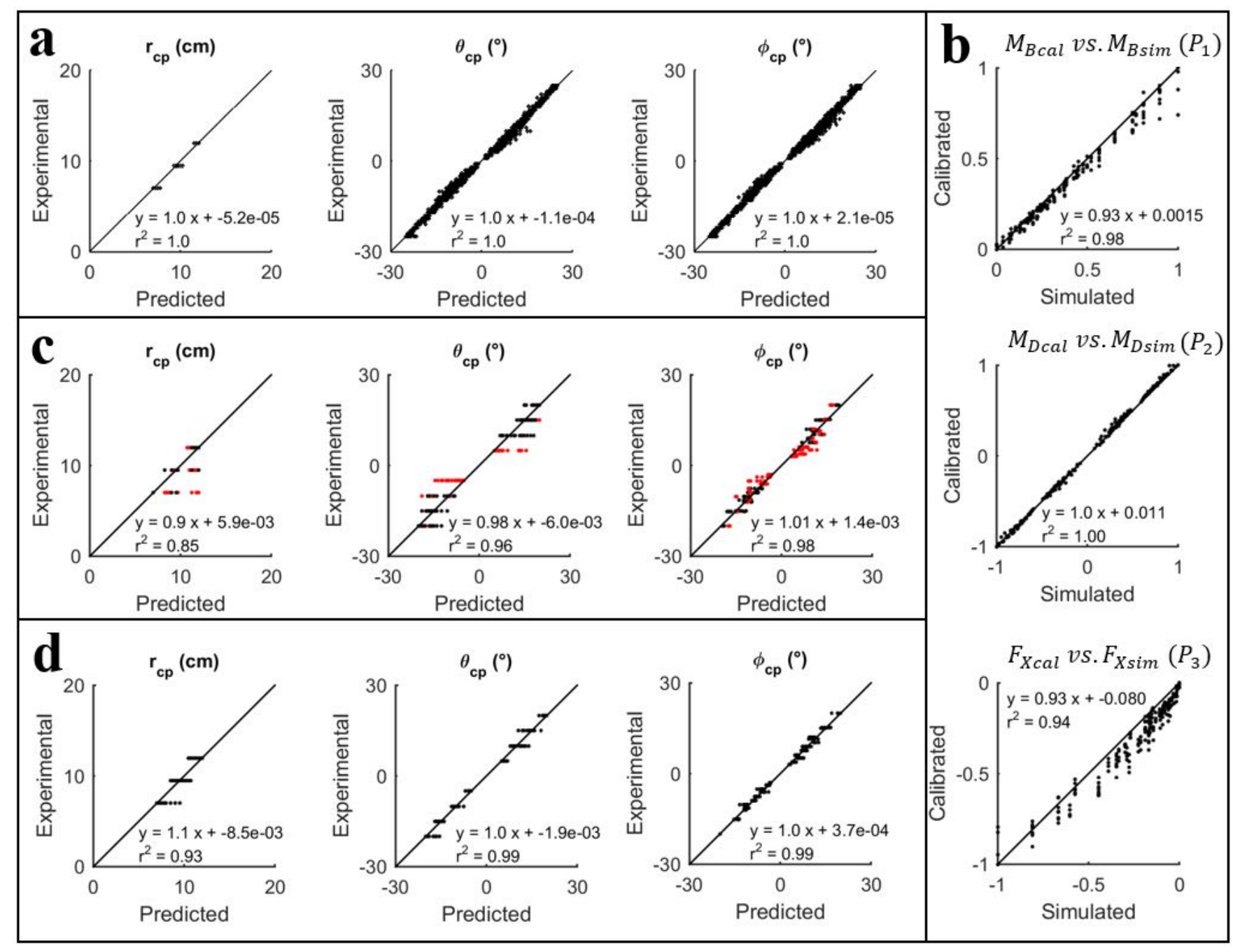

Fig. 4. Estimation of 3D contact point location with and without the use of simulations: (a) Simulations confirm that a whisker with the geometry chosen for the robotic whisker can generate a unique mapping between $\left[M_{B}, M_{D}, F_{X}\right]$ and $\left[r_{c p}, \theta_{c p}, \phi_{c p}\right]$ over the desired range. The simulated dataset consists of 7,500 data points. The coefficient of determination for all contact points $\left[r_{c p}, \theta_{c p}, \phi_{c p}\right]$ is statistically indistinguishable from 1.0. (b) The hardware follicle can be linearly calibrated to yield a good match to simulation results, but larger error is observed for larger angle deflections. All signals have been normalized according to the procedures described in Sec. III (c) Contact point estimation using calibrated experimental voltages (mappings generated from eqs. 67 The calibrated experimental contact point coordinate is plotted as a function of the predicted point coordinate. The calibrated experimental contact point coordinate is plotted as a function of the predicted point coordinate. Contact points with angular deflections less than $10^{\circ}$ are plotted in red and have been omitted from the regression calculation. This cutoff angle effectively represents the "dead-band" zone for the sensor, below which signals did not reliably match simulation. (d) Contact point estimation using un-calibrated experimental voltages. The experimental contact point coordinate is plotted as a function of the predicted contact point. No points are excluded.

\section{E. $3 D$ contact point estimation without calibration}

The method for contact point determination shown in Fig. $4(\mathrm{c})$ has only moderate accuracy and is time consuming. If we were to use this method, each whisker in a multi-whisker robotic system would have to be calibrated based on simulation data specific to the geometry of that whisker.

We therefore performed a set of experiments in which we used only the normalized voltage signals from the strain gages to compute contact point location. As described in Sec. II] we developed a mapping between the four voltages from the sensor and the 3D contact point. Note that this method still requires use of the experimental set-up in Fig. 2 but does not require any simulations.

The normalized output voltages $\left(V_{1}\right.$ to $\left.V_{4}\right)$ achieved coeffi- cients of determination of $0.967,0.996$, and 0.997 for the three contact point coordinates $\left(r_{c p}, \theta_{c p}\right.$ and $\left.\phi_{c p}\right)$ during training. This result led us to predict that these normalized sensor outputs should be sufficient to predict contact point location without the need for calibration and simulation. This prediction is confirmed in Fig. 4(d), which shows the output of the random forest regression on the normalized output voltages over the full dataset. The coefficients of determination are above 0.93 for all contact point locations. The cross-validation for this dataset was 0.838 .

Notice that although the $r^{2}$ values are higher for the results shown in Fig. 4(c) (calibrated experimental data), this figure shows regression only over a subset of the data. Higher accuracy could likely be achieved with more data. Fig. 4(d), 
in contrast, has no points omitted and suggests that greater resolution can be achieved in less time.

Further analysis showed that to achieve errors less than 1.5 $\mathrm{cm}$, only $3.6 \%$ of the experimental data set would need to be omitted. In fact, $78 \%$ percent of the data predicted the contact point with an accuracy of $0.6 \mathrm{~cm}$.

Interestingly, the maximum sensor error did not occur at particular values of radial distance or angle, indicating that the sensor itself has its own unique mapping between the mechanical signals at the base and contact point location.

It should be noted that each contact point was only tested once in the experimental set-up. This scarcity of examples amplifies any errors that occurred during testing and can bias the outcome of all predictions, causing lower correlations overall and lower cross-validation scores.

F. Contour extraction using continuous sweeps of the whisker

Given the success of the results shown in Fig. 4(d), we tested the ability of the senor to perform 3D contour extraction on a cylinder and a cone. The sensor was rotated by hand against the two objects. As described in Sec. III the sensor was fixed so that it could only rotate about the $\mathrm{z}$-axis during this experiment, but the cone shape was deliberately chosen to ensure "slip" of the whisker on the object.

At each point in time, the 3D contact point relative to the whisker base was determined using eq. 8 . Determination of the 3D contact point required no information other than $V_{1}$ to $V_{4}$ obtained directly from the whisker base. To convert the 3D contact point to the laboratory frame, an encoder was attached to the base of the whisker. We emphasize that information from the encoder was used only to convert the 3D contact points to the laboratory frame, and not used for contact point determination. Fig. 5(a) shows the results in whisker-centered coordinates obtained during two sweeps of the whisker against a cone, with the contact point coordinates plotted as a function of encoder position. As the encoder angle increases, $r_{c p}$ decreases as the contact point nears the base of the cone. The magnitudes of both $\theta_{c p}$ and $\phi_{c p}$ increase as the whisker is forced to deflect further against the cone. The sign for $\theta_{c p}$ is negative because in whisker-centered coordinates as $\theta_{\text {enc }}$ increases, the whisker is increasingly deflected in the negative y-direction, corresponding to a negative $\theta_{c p}$. The sign for $\phi_{c p}$ is positive because the whisker is increasingly slipping up the cone.

Using eq. 8 , the contact point locations in Fig. 5 (a) were converted to the laboratory frame. The ability of the whisker to extract the contour of a shape is shown in Fig. 5(b), which superimposes the contact points converted to the laboratory frame on top of the known shape and location of a cylinder and cone. In both examples, the contact point locations predicted by the sensor trace out the 3D edges of the shapes. Videos of Fig. 5.b) are shown in supplementary video 1 and 2 (located at github.com/SeNSE-lab). Further trials of the sensor could in the future map out the full surface.

It should be noted that in Fig. 5(a), the predicted radial distance is constant at both the beginning and end of the trial while the encoder angle is increasing. This effect occurs because the whisker is impacting outside of the range of motion tested and is discussed further in Sec. IV This effect also occurs when the angular deflection is zero.
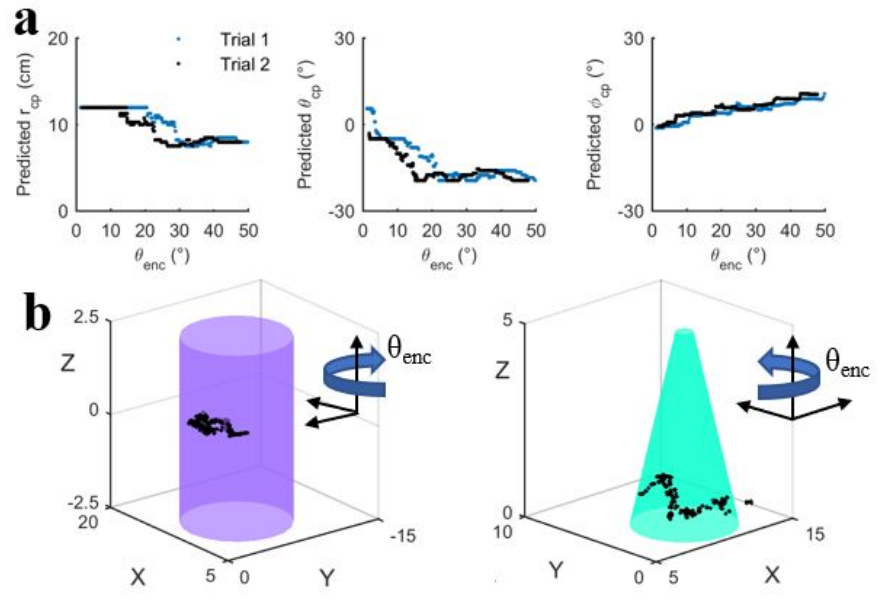

Fig. 5. Contour extraction with the follicle-whisker sensor: (a) Two sweeps of the sensor deflecting against a cone. The sensor can correctly identify decreasing radial distance, decreasing angle of deflection and increasing angle of elevation (b) 3D contact points obtained during two sweeps of the whisker against two different objects (a cylinder and a cone) are converted to the laboratory frame and superimposed on the known coordinates of a cylinder (left) and a cone (right). The whisker was deflected by hand against the cylinder in a clockwise direction and against the cone in a counterclockwise direction. The sensor is located at the origin.

\section{DisCUSSION AND CONCLUSION}

Several previous studies have used rates of change of bending moment at the base of the whisker to determine contact point location [7-14]. The present approach is novel in that it statically determines the 3D contact point location at each instant of time, and does not depend on rates of change. In other words, determining the 3D whisker-object contact point no longer depends on the whisker's trajectory (history). A whisker's trajectory along a surface is strongly influenced by friction, texture, surface defects, and local slope: the present work offers an approach to reduce the influence of these variables for contour extraction. Rates of change in bending moment could then be used to sense object motion or object compliance [6, 29, 30].

The hardware whisker employed in the present work is a simplified version of a biological rat whisker: parameters were limited to arc length, base diameter, tip diameter, and Young's modulus. A follicle-like sensor was developed to measure signals related to the two bending moments $\left(M_{Y}\right.$ and $\left.M_{Z}\right)$ and the axial force $\left(F_{X}\right)$. The two bending moments were later used to compute $M_{B}$ and $M_{D}$ during the analysis. Although $M_{Y}$ and $M_{Z}$ could have been used as two predictors directly, $M_{B}$ and $M_{D}$ were chosen because they are more intuitive and were shown to be part of a unique triplet in Huet et al., 2017.

We then used simulations to verify that the geometry chosen for our hardware whisker had a unique mapping between the mechanical signals at the base of the whisker and the 3D contact point coordinates. An important feature of the 
hardware whisker geometry was its taper. Previous studies have suggested that the primary importance of whisker taper is that it alters the angle at which a whisker will slip off an object [22, 23]. We suggest that an equally important reason is that taper is essential for producing unique mappings between mechanical signals at the whisker base and the 3D contact point [6, 30].

The signals generated from the hardware whisker were then tested against simulation results. The simulations were performed using software that assumes zero friction and solves for the normal force at the contact point to determine the mechanical signals at the whisker base [6, 31, 32]. These simulations directly guided our choice of predictor signals. Thus, $P_{1}$ and $P_{2}$ were selected to model $M_{B}$ and $M_{D}$, because these signals directly determine the whisker's angular deflection. The third predictor, $P_{3}$, was chosen to approximate the axial force, because $M_{B}$ and $M_{D}$ alone are not sufficient to estimate the radial distance of contact. Even though $P_{3}$ was found to be a relatively poor match to simulated axial force (Fig. 4(b)), a relatively good estimate for radial distance was achieved, provided signals were outside the sensor's "deadband."

As suggested by Table 2 of Huet et al., 2017, similar results could potentially be achieved with many other choices for the predictor variables, especially if large angle deflections are excluded. Even further, new predictor variables could be assembled from our mechanical signals that have no clear mechanical interpretation, so long as they can accurately predict the output variables.

We next tested two methods for 3D contact point determination in hardware. Satisfactory estimates of contact point location were obtained by calibrating the experimental data to simulations, but results improved greatly when the normalized voltages $V_{1}$ to $V_{4}$ were used directly. This improvement indicates that time-consuming calibration is not required to accurately determine contact point location, and also suggests that this approach could be used for continuous contour extraction, as shown in Fig. 5. Results of Fig. 5 confirm that the voltage signals at the whisker base can be used to trace out the contour of two different shapes. We fully anticipate that additional sweeps of the whisker could map out complete object surfaces.

An important source of error in the contours shown in Fig. 5 is that multi-output random forest regression was used to create mappings between 3D contact point locations and mechanical signals. Importantly, multi-output regression is inherently poor at generalizing beyond the trained range of motion, and we only sampled a $\sim 8 \mathrm{~cm}$ region along the length of the whisker. This limited training range had a particularly noticeable effect on predictions for the radial contact coordinate shown in Fig. 5(a). Although the whisker almost certainly made contact at a radial distance larger than $12 \mathrm{~cm}$ at the beginning of the sweep, the algorithm continuously predicted impact at $12 \mathrm{~cm}$ even while the encoder angle was increasing. We fully expect this effect could be mitigated by including a larger range of motion in the training set. Otherwise, algorithms better suited to generalize could be used to accurately extrapolate to new data.

Another large source of error stems from those deflections with small angles (below $10^{\circ}$ ). These deflections were shown to have the largest percent difference as compared to simulated data. These contributed a large source of error during calibration and as such should be defined as a potential "deadband" zone for the sensor and accounted for during further experiments.

Using artificial whiskers as sensors on robotic platforms has potential utility in many engineering applications. Whiskers could provide spatial mappings in environments where classical sensory systems fail, such as in the dark or in partially occluded spaces, murky waters, or sand. Previous whiskerbased tactile systems have taken many forms,

Previous whisker based tactile systems have taken many forms, including mobile robots with full whisker arrays [8, 3335, 38], single whisker position sensors [7, 8, 28, 39, 40, 42] and as sensors to measure the texture of an object [34, 36, 37]. Sensing mechanisms have ranged from strain gages [33, 37 38] to load cells [39, 40] to Hall effect sensors [8, 14, 41] to torque sensors [7, 42].

The relative simplicity of the whisker sensors developed here means that they are well-suited for use in remote environments, with new mappings being learned "on-the-fly" in the case of damage or environmental changes. Applications could include exploration in remote areas including underwater or even space. Future work will focus on active sensing (c.f. [35]) and on developing a platform to enable complete mapping of 3D spaces.

This system also has implications for the study of biological sensing. The biological rat whisker is so tiny, and has such a large length to diameter ratio, that it is difficult to experimentally manipulate and to simulate. By building a biomimetic scaled model of a rat whisker, we can systematically explore the mechanical space and experimentally test conditions in which simulations tend to fail (e.g., friction, noise, nonstationarities, and air turbulence). We specifically anticipate using similar hardware models to improve our understanding of the encoding properties of neurons in the vibrissaltrigeminal system.

\section{ACKNOWLEDGMENTS}

This multi-year research effort was supported by a sequence of awards from the National Science Foundation (NSF): award numbers EFRI-0938007, IIS-1208118, and BCS-1734981 to MJZH. This material is based upon work supported by the National Science Foundation Graduate Research Fellowship Program under Grant No. DGE-1324585 to HME. We thank Anne E.T. Yang for her help with the figures and many useful discussions, the Brinson Group at Duke University and specifically David Collinson for fabricating the whiskers, and Mikhail Todes and Kegan Leizerman for their early contributions to the design of the artificial follicle. 


\section{REFERENCES}

[1] E. Guić-Robles, C Valdivieso, and G Guajardo. Rats can learn a roughness discrimination using only their vibrissal system. Behavioural Brain Research, 31(3):285-289, 1989.

[2] GE E Carvell and DJ J Simons. Biometric analyses of vibrissal tactile discrimination in the rat. The Journal of Neuroscience, 10(8):2638-2648, 1990.

[3] R. A. Grant, S. Haidarliu, N. J. Kennerley, and T. J. Prescott. The evolution of active vibrissal sensing in mammals: evidence from vibrissal musculature and function in the marsupial opossum Monodelphis domestica. Journal of Experimental Biology, 216(18):3483-3494, 2013.

[4] S. B. Vincent. The functions of the vibrissae in the behavior of the white rat (1912). Behav. Monogr., 1(1-81).

[5] Satomi Ebara, Kenzo Kumamoto, Tadao Matsuura, Joseph E. Mazurkiewicz, and Frank L. Rice. Similarities and differences in the innervation of mystacial vibrissal follicle-sinus complexes in the rat and cat: A confocal microscopic study. Journal of Comparative Neurology, 449(2):103-119, 2002.

[6] Lucie A Huet, John W Rudnicki, and Mitra J.Z. Hartmann. Tactile Sensing with Whiskers of Various Shapes: Determining the Three-Dimensional Location of Object Contact Based on Mechanical Signals at the Whisker Base. Soft Robotics, 4(2):88102, 2017.

[7] Makoto Kaneko, Naoki Kanayama, and Toshio Tsuji. Active antenna for contact sensing. IEEE Transactions on Robotics and Automation, 14(2):278-291, 1998.

[8] Dae Eun Kim and Ralf Möller. Biomimetic whiskers for shape recognition. Robotics and Autonomous Systems, 55(3):229-243, 2007.

[9] Joseph H Solomon and Mitra J Hartmann. Biomechanics: robotic whiskers used to sense features. Nature, 443(7111):525, 2006.

[10] Knarik Bagdasarian, Marcin Szwed, Per Magne Knutsen, Dudi Deutsch, Dori Derdikman, Maciej Pietr, Erez Simony, and Ehud Ahissar. Pre-neuronal morphological processing of object location by individual whiskers. Nature neuroscience, 16(5):622-31, 2013.

[11] Joseph H. Solomon and Mitra J.Z. Hartmann. Artificial whiskers suitable for array implementation: Accounting for lateral slip and surface friction. IEEE Transactions on Robotics, 24(5):1157-1167, 2008.

[12] Joseph H Solomon and Mitra J Z Hartmann. Extracting Object Contours with the Sweep of a Robotic Whisker Using Torque Information. The International Journal of Robotics Research, 29(9):1233-1245, 2010.

[13] J Alexander Birdwell, Joseph H Solomon, Montakan Thajchayapong, Michael a Taylor, Matthew Cheely, R Blythe Towal, Jorg Conradt, and Mitra J Z Hartmann. Biomechanical models for radial distance determination by the rat vibrissal system. Journal of neurophysiology, 98(4):2439-2455, 2007.

[14] Mathew H Evans, Charles W Fox, Nathan F Lepora, Martin J Pearson, J Charles Sullivan, and Tony J Prescott. The effect of whisker movement on radial distance estimation: a case study in comparative robotics. Frontiers in Neurorobotics, 6:12, 2013.

[15] Vahid Reza Adineh, Boyin Liu, Ramesh Rajan, Wenyi Yan, and Jing Fu. Multidimensional characterisation of biomechanical structures by combining Atomic Force Microscopy and Focused Ion Beam: A study of the rat whisker. Acta Biomaterialia, 21:132-141, 2015 .

[16] O. F. Chernova and V. F. Kulikov. Structural differences between the shafts of mammalian vibrissae and hairs and their causes. Doklady Biological Sciences, 438(1):182-185, 2011.

[17] Kathrin Carl, Wolfram Hild, Jörg Mämpel, Cornelius Schilling, René Uhlig, and Hartmut Witte. Characterization of statical properties of rat's whisker system. IEEE Sensors Journal, 12(2):340-349, 2012.
[18] Danja Voges, Kathrin Carl, Gertrud J. Klauer, René Uhlig, Cornelius Schilling, Carsten Behn, and Hartmut Witte. Structural characterization of the whisker system of the rat. IEEE Sensors Journal, 12(2):332-339, 2012.

[19] Brian W Quist, Rafay A Faruqi, and Mitra J Z Hartmann. Variation in Young's modulus along the length of a rat vibrissa. Journal of Biomechanics, 44(16):2775-2781, 2011.

[20] E. K. Herzog, D. F. Bahr, C. D. Richards, R. F. Richards, and D. M. Rector. Spatially Dependent Mechanical Properties Of Rat Whiskers For Tactile Sensing. MRS Proceedings, 844:Y3.6/R3.6, 2004.

[21] L Ibrahim and E A Wright. The growth of rats and mice vibrissae under normal and some abnormal conditions. Journal of embryology and experimental morphology, 33(4):831-44, 1975.

[22] Samuel Andrew Hires, Lorenz Pammer, Karel Svoboda, and David Golomb. Tapered whiskers are required for active tactile sensation. eLife, 2013(2):e01350, 2013.

[23] Christopher M Williams and Eric M Kramer. The advantages of a tapered whisker. PLoS ONE, 5(1), 2010.

[24] Sejoon Ahn and Dae Eun Kim. Radial distance estimation with tapered whisker sensors. Sensors (Switzerland), 17(7):1659, 2017.

[25] Hayley M Belli, Anne E T Yang, Chris S Bresee, and Mitra J. Z. Hartmann. Variations in vibrissal geometry across the rat mystacial pad: base diameter, medulla, and taper. Journal of Neurophysiology, 117(4):1807-1820, 2017.

[26] Jeffrey D. Moore, Nicole Mercer Lindsay, Martin Deschênes, and David Kleinfeld. Vibrissa Self-Motion and Touch Are Reliably Encoded along the Same Somatosensory Pathway from Brainstem through Thalamus. PLoS Biology, 13(9):e1002253, 2015.

[27] Heather R. Beem and Michael S. Triantafyllou. Wakeinduced slaloming' response explains exquisite sensitivity of seal whisker-like sensors. Journal of Fluid Mechanics, 783(2015):306-322, 2015.

[28] Ozkan Bebek and M. Cenk Cavusoglu. Whisker sensor design for three dimensional position measurement in robotic assisted beating heart surgery. IEEE International Conference on Robotics and Automation, (April):225-231, 2007.

[29] Lorenz Pammer, Daniel H O'Connor, S Andrew Hires, Nathan G Clack, Daniel Huber, Eugene W Myers, and Karel Svoboda. The Mechanical Variables Underlying Object Localization along the Axis of the Whisker. Journal of Neuroscience, 33(16):6726-6741, 2013.

[30] J. H. Solomon and M. J. Z. Hartmann. Radial distance determination in the rat vibrissal system and the effects of Weber's law. Philosophical Transactions of the Royal Society B: Biological Sciences, 366(1581):3049-3057, 2011.

[31] Lucie A Huet, Christopher L Schroeder, and Mitra J Z Hartmann. Tactile signals transmitted by the vibrissa during active whisking behavior. Journal of Neurophysiology, 113(10):35113518, 2015.

[32] Lucie A Huet and Mitra J.Z. Hartmann. Simulations of a Vibrissa Slipping along a Straight Edge and an Analysis of Frictional Effects during Whisking. IEEE Transactions on Haptics, 9(2):158-169, 2016.

[33] Ben Mitchinson, J. Charles Sullivan, Martin J. Pearson, Anthony G. Pipe, and Tony J. Prescott. Perception of simple stimuli using sparse data from a tactile whisker array. Lecture Notes in Computer Science, 8064 LNAI:179-190, 2013.

[34] Anil K Seth, Jeffrey L McKinstry, Gerald M Edelman, and Jeffrey L Krichmar. Spatiotemporal processing of whisker input supports texture discrimination by a brain-based device. In Animals to Animats 8: Proceedings of the Eighth International Conference on the Simulation of Adaptive Behavior. Meyer, JA (ed.) MIT Press, Cambridge, MA, pages 130-139, 2004. 
[35] J. Charles Sullivan, Ben Mitchinson, Martin J. Pearson, Mat Evans, Nathan F. Lepora, Charles W. Fox, Chris Melhuish, and Tony J. Prescott. Tactile discrimination using active whisker sensors. IEEE Sensors Journal, 12(2):350-362, 2012.

[36] M Fend, Simon Bovet, H Yokoi, and R Pfeifer. An active artificial whisker array for texture discrimination. Proc. IEEE/RSJ Int. Conf. Intelligent Robots and Systems (IROS 2003), 2:10441049, 2003.

[37] Charles W Fox, Ben Mitchinson, Martin J Pearson, Anthony G Pipe, and Tony J Prescott. Contact type dependency of texture classification in a whiskered mobile robot. Autonomous Robots, 26(4):223-239, 2009.

[38] Christopher L. Schroeder and Mitra J Z Hartmann. Sensory prediction on a whiskered robot: A tactile analogy to "optical flow". Frontiers in Neurorobotics, 6:1-11, 2012.

[39] Gregory R. Scholz and Christopher D. Rahn. Profile sensing with an actuated whisker. IEEE Transactions on Robotics and Automation, 20(1):124-127, 2004.

[40] Tyler N Clements and Christopher D Rahn. Three-Dimensional Contact Imaging With an Actuated Whisker. IEEE Transactions on Robotics, 22(4), 2006.

[41] Dae Eun Kim and Ralf Möller. Biomimetic Whisker Experiments for Tactile Perception. Proceedings of Int. Symposium on Adaptive Motion, pages 1-7, 2005.

[42] Naohiro Ueno, Mikhail M. Svinin, and Makoto Kaneko. Dynamic contact sensing by flexible beam. IEEE/ASME Transactions on Mechatronics, 3(4):254-264, 1998. 